\title{
Characterization of the electrical and optical properties of a gliding arc tornado device
}

\author{
R. Barni ${ }^{1, a}$, P. Alex ${ }^{1}$, A. Salanti ${ }^{1}$, C. Canevali ${ }^{2}$, L. Zoia ${ }^{3}$, M. Orlandi ${ }^{3}$, and C. Riccardi ${ }^{1, b}$ \\ 1 Dipartimento di Fisica G.Occhialini, Università degli Studi di Milano-Bicocca, piazza delle Scienza 3, 20126 Milan, Italy \\ 2 Dipartimento di Scienza dei Materiali, Università degli Studi di Milano-Bicocca, Milan, Italy \\ 3 Dipartimento di Scienze dell'Ambiente e della Terra, Università degli Studi di Milano-Bicocca, Milan, Italy
}

Received 14 October 2020 / Accepted 10 March 2021 / Published online 3 May 2021

(C) The Author(s) 2021

\begin{abstract}
Optical emission spectroscopy was used to characterize the gas phase in a gliding arc tornado reactor used for plasma applications. Results concerning the transition between spark and fully developed arc regimes are presented, by comparing with the electrical characteristics of the discharge. An extended analysis of spectra of air and helium discharges mixed with water and hexamethyldisiloxane vapors was performed, with the identification of active radical species involved in the surface functionalization of polymeric nanoparticles.
\end{abstract}

\section{Introduction}

The gliding arc tornado (GAT) was proposed long ago to improve the properties of gliding arcs reactors, in particular to insure a better insulation of the device walls from the discharge, which could reach a higher level of non-equilibrium and much larger residence times to boost the reactivity [1]. These devices were mostly used for industrial applications including fuel conversion, carbon dioxide conversion and waste treatment $[2,3]$. Their name, GAT, refers to the formation of a reverse vortex flow configuration, a tornado, usually achieved by tangential gas injection near the walls in a cylindrical chamber. We have developed and used a kind of these devices for the treatment of lignin powder by plasmas [4]. Milled lignin can be easily injected in a sustained gas flux, and particles are advected in the hydrodynamical flow. So optimal interaction with the discharge gas phase could be achieved. Lignin surface functionalization is critical to transform this waste product into a renewable resource that could be used in industry, for instance, as a bio-polymer [5]. We consider we could achieve a suitable functionalization of lignin with plasma processing using our GAT device [6,7]. Here, we will present the results of an experimental campaign aimed to the characterization of the electric discharges and the plasma processing in the device, based on electrical and optical diagnostics. Optical Emission Spectroscopy (OES) is a suitable technique to investigate the plasma gas phase, since it is passive and non-perturbing, and it takes advantage of

\footnotetext{
a e-mail: ruggero.barni@mib.infn.it (corresponding author)

b e-mail: claudia.riccardi@unimib.it
}

the rich emission pattern in/near visible range of such systems [8-10]. Besides gathering information about the discharge, it is possible to get insight on the interactions of lignin particles with plasma and the mechanism of their surface treatment. In particular, we have studied the effects of the addition of water and hexamethyldisiloxane (HMDSO) vapors to the helium flow used to fed the tornado discharge [11]. We demonstrate that, in the device, it was possible to achieve a substantial dissociation of the vapors and a grafting of radicals on the lignin particles flown through the discharge.

\section{Experimental setup}

The discharge chamber consists of a pyrex tube (39 $\mathrm{mm}$ inner diameter, $2.5 \mathrm{~mm}$ thick, $200 \mathrm{~mm}$ long), closed at one side by a Teflon cylinder cap, through which the electrode wire enters. On the other side, the tube is partially closed by an hollow stainless-steel disk ( 25 $\mathrm{mm}$ hole diameter, $8 \mathrm{~mm}$ thick). Gas inlet and sealing are provided by a couple of Teflon hollow disks surrounding the pyrex tube. The chamber has an effective height of $55 \mathrm{~mm}$ (volume about $60 \mathrm{~cm}^{3}$ ). Gas is injected tangentially by two opposite channels with square cross section $\left(3 \times 3 \mathrm{~mm}^{2}\right)$ excavated in one of the Teflon disks. A reverse flow vortex is produced in the chamber spiraling downward along the pyrex inner surface until it reaches the back cap and a jet is formed along the axis exiting from the front disk central hole. Using a compressed air supply with one atmosphere over-pressure, the effective conductance was estimated at about 20 $\mathrm{L} / \mathrm{s}$, corresponding to a residence time in the chamber of about $3 \mathrm{~ms}$ and an outflow velocity of about 45 


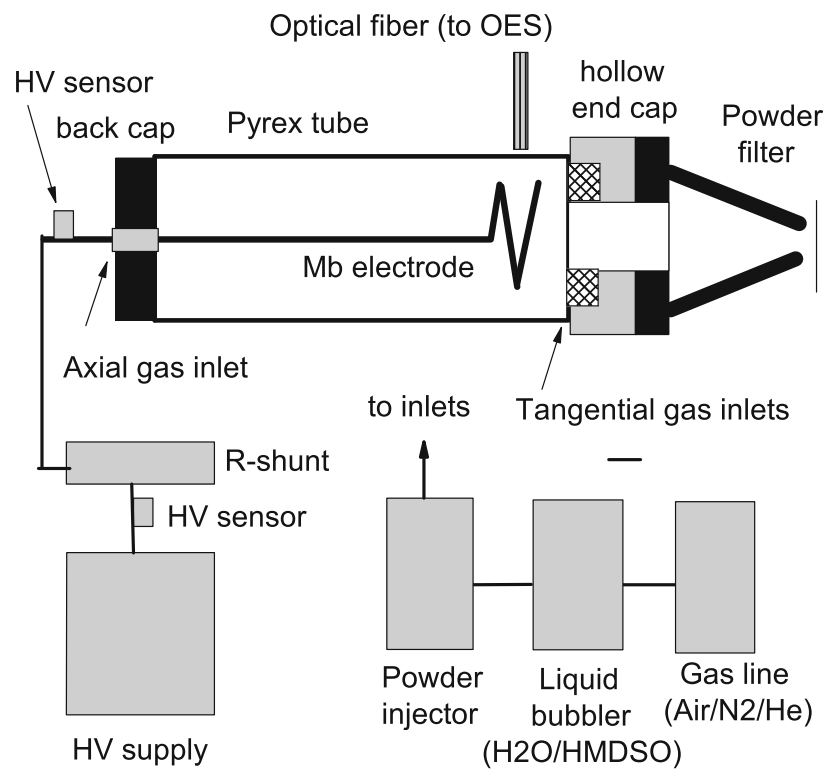

Fig. 1 The experimental setup of the GAT device

$\mathrm{m} / \mathrm{s}$. Outside the chamber, the outgoing flow is guided through a conical nozzle $(30 \mathrm{~mm}$ height, final orifice 24 $\mathrm{mm}$ diameter), to which further piping elements could be added, to recover nanoparticles or to prevent arcing reaching outside for instance. The tornado could be imaged by using smoke or tracing particles in the inlet flow. Air rotates backward elicoidally along the pyrex surface, up to the Teflon cap, and exits longitudinally as a jet mostly aligned along the axis. The discharge was ignited and supplied by means of a highvoltage DC generator (SHV9000 by Alintel). Its performances cannot exceed $5 \mathrm{kV}$ and $2 \mathrm{~A}$ at most. The instruments itself provided a few integrated measurements, for instance, the total charge effectively delivered and the mean applied high voltage, which could be used to estimate the actual power absorbed by the device. During operation, besides a maximum HV-level setting, the generator is regulated by a variable mean current intensity level. Its value effectively controls the actual electrical characteristics of the discharge, as we will see in the following. The supply was connected to the anode electrode through an electrical circuit (a set of 16 high-voltage, high power resistances, with a global resistance of $4 \mathrm{k} \Omega$ ), which helps to control the electrical characteristics of the discharges, avoiding short circuitry. HV sensors were also inserted in the circuit to measure the instantaneous values of the voltages on a digital scope. A layout of the experimental setup is displayed in Fig. 1. Due to both the limitation in the output current provided by the generator and the external circuit impedance, the discharge assumes an intermittent spark character at low current settings [12]. It develops to a full arcing above a certain threshold of the current set, slightly depending on the inlet flow pressure and gas mixture composition. More specifically, a three-turn molybdenum electrode acts as the arc anode, as shown in Fig. 2. HV sensors measure the

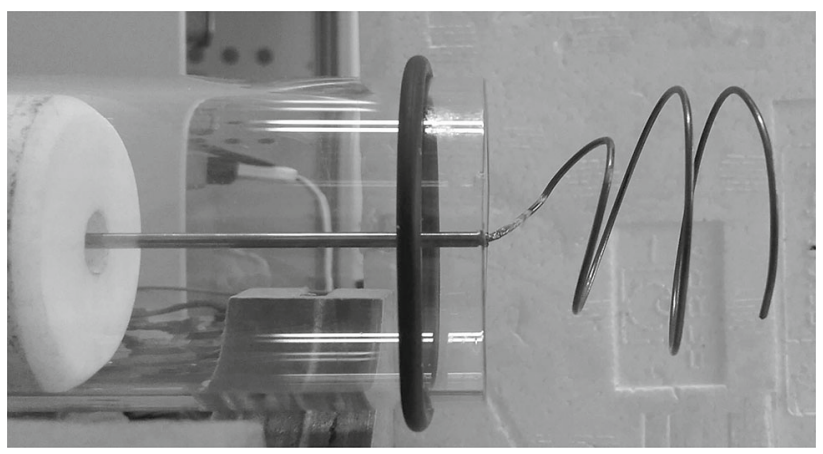

Fig. 2 The GAT anode electrode

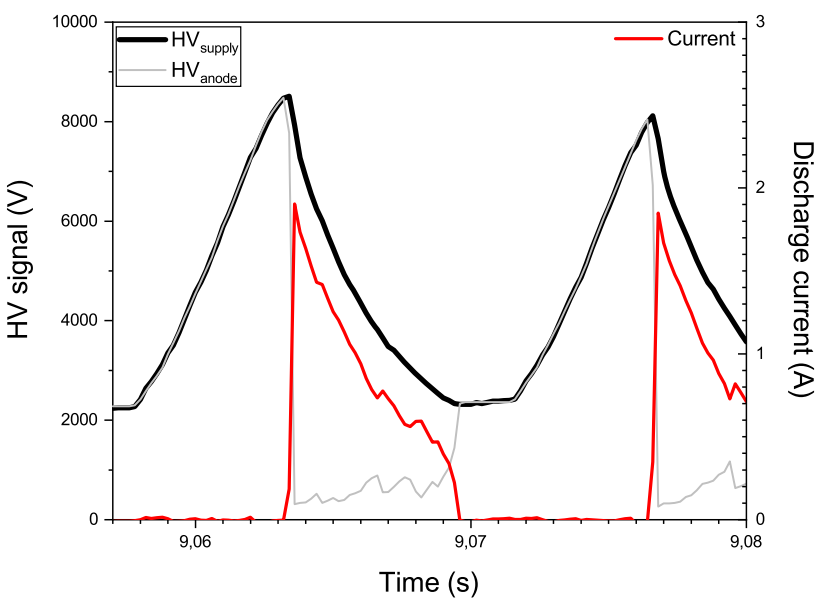

Fig. 3 HV signals and the calculated discharge current

instantaneous voltage at the ends of the resistors set and thus can be used to estimate also the instantaneous value of the discharge current, as it is shown in Fig. 3. It could be grasped as the system shows intense current bursts with a limited duration, separated by dead times, corresponding to a sequence of spark discharges. Although not exactly constant, the shape, the amplitude, the duration, and the repetition rate of bursts were comparable and fairly cyclic. These parameters were measured and averaged using long time series $\left(10^{7}\right.$ samples) with a digital scope, and their statistical properties were studied.

\section{Electrical characteristics}

The electrical characteristics of a typical spark discharge are shown in Fig. 3. The voltage measured by the two HV sensors at the ends of the shunt resistance is used to calculate the discharge current and overall impedance. It is clear that whenever the anode voltage exceeds a threshold value, the discharge gets ignited and the anode voltage decrease to a lower, quite small and somewhat constant, level. The discharge arc evolves, and the electric current has a somewhat trapezoidal shape, decreasing steadily after the breakdown. After a few milliseconds, the discharge is over and the elec- 


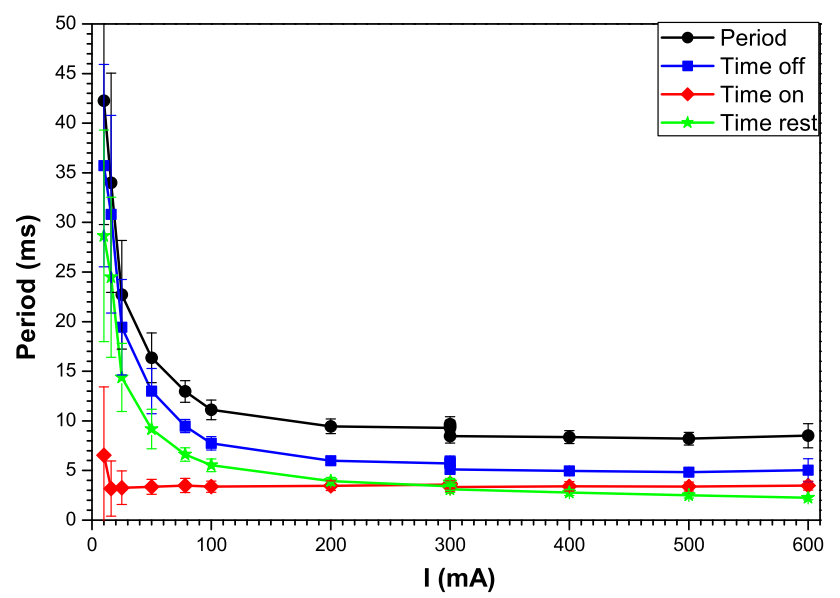

Fig. 4 Mean durations of discharge and its phases as a function of the operating current

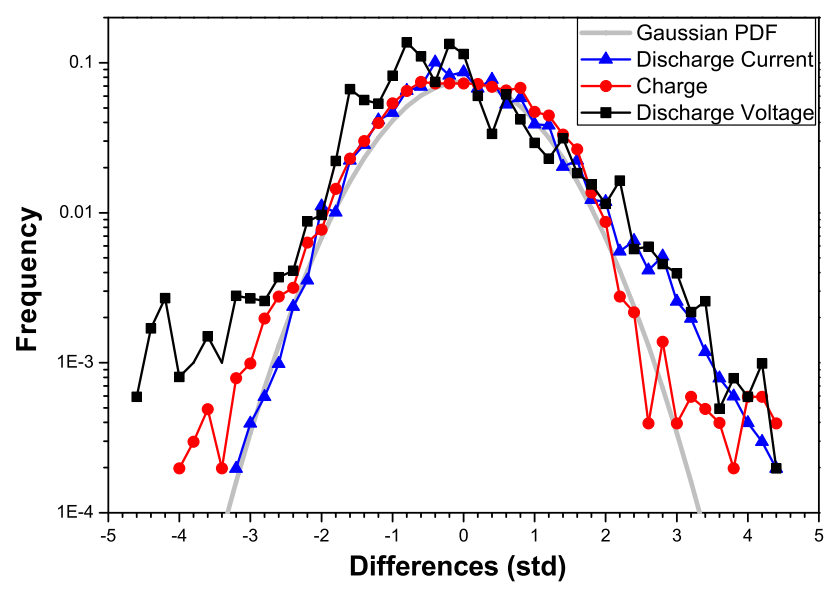

Fig. 5 PDF of discharge electrical characteristics, normalized to their standard deviations

tric current drops to zero. A quiet period at a voltage level lower than the threshold is followed by a slow voltage increase, until the threshold is exceeded again. The discharging is repeated with a roughly constant frequency. The current peak amplitudes and durations are roughly the same, within a not negligible spread, reflecting the individual characteristics of each discharge. In particular, we observe that discharge could be operated even at very low current settings of $10 \mathrm{~mA}$, whereas transition to a steady arc discharge happens above 600 mA. As it could be grasped from Fig. 4, the prescribed mean current absorption by the supply is obtained by increasing the mean period between subsequent sparkings. Rather than through a tuning of the output voltage, leading to a modification in the discharge current or average duration, this is reached by increasing the off time between sparks. This happens mostly by a growth of the rest period, after the discharge quenching, when the output voltage is kept at a suitable level below ignition threshold. The distribution of individual discharge characteristics, (the probability distribution functions, PDF, shown in Figs. 5 and 6), clearly demonstrates that

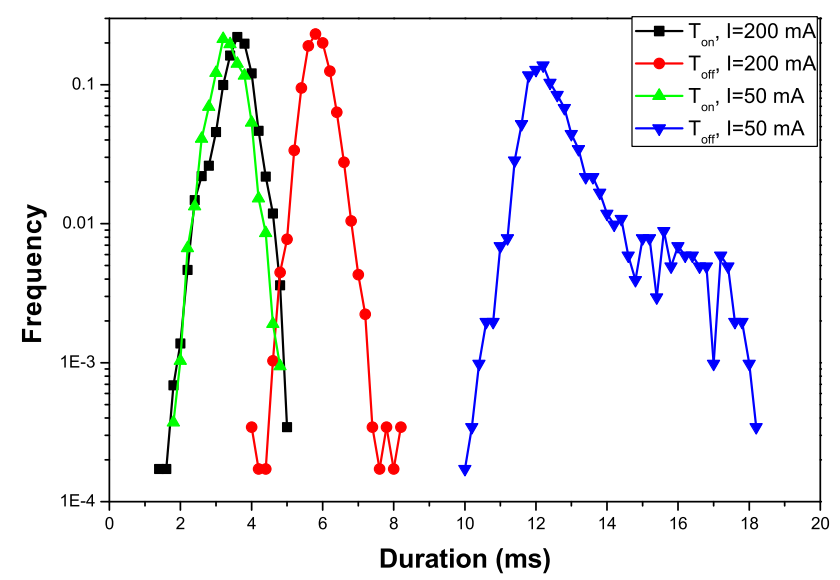

Fig. 6 PDF of discharge durations, for operating current of 25 and $200 \mathrm{~mA}$

the setting control does not act on the single discharge event, which shows a substantial range of variations, not depending purely on measure precision. In particular, they appear to be quiet skewed with fat tails, respect to expected Gaussian distribution [13,14]. A detailed statistical analysis of such distributions exceeds the limited scope of our paper, but it is interesting to pursue, and it could reveal aspects of the ignition mechanism and of its repetition [15].

\section{Optical emission spectroscopy}

While the pattern of the single discharge is somewhat fuzzy, it appears that on average, most of the flow cross section is affected by the discharge and emits light. OES of the discharge is used to gain insight in the excited species produced. Measurements of the emission spectra are obtained with a wide-band, low-resolution spectrometer. The spectrometer (PS2000 by Ocean Optics) has a resolution of about $0.4 \mathrm{~nm}$ and a spectral band extending from 180 to $860 \mathrm{~nm}$. It is equipped with a 10 $\mu \mathrm{m}$ slit, a holographic grating (600 lines/mm, blazed at $400 \mathrm{~nm}$ ) and a coated quartz lens to increase sensitivity in UV [12]. Emission line intensity was measured, and atoms and molecules have been identified $[16,17]$. The light emitted from the plasma sheet was collected by means of an UV-enhanced optical fiber placed just in front of the exit $10 \mathrm{~cm}$ apart, to not perturb the outflowing gas jet. The view field of the optical fiber was large enough to cover the whole light-emitting region and to smear possible discharge disuniformities. A typical spectrum is shown in Fig. 7. Spectra of the light emitted from the discharge show a broad continuum peaking at about 500-550 nm, with a rich superimposed structure of lines, some quite narrow, comparable to the spectrometer resolution, often in multiplets, and other that appears larger, clearly emitted by molecular states and appearing together due to their unresolved rotational substructure. As the mean current setting is increased, the continuum contribution increases at expenses of the 


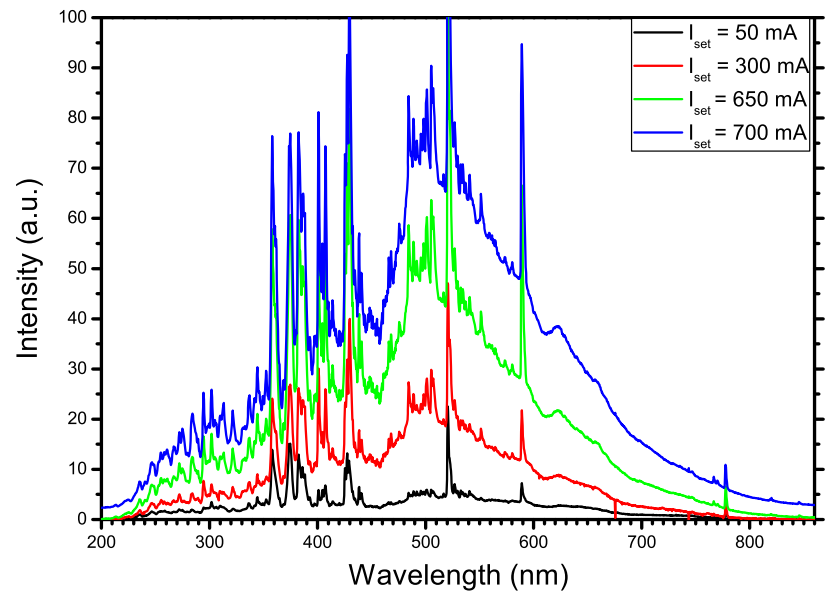

Fig. 7 A typical spectrum of the GAT discharge

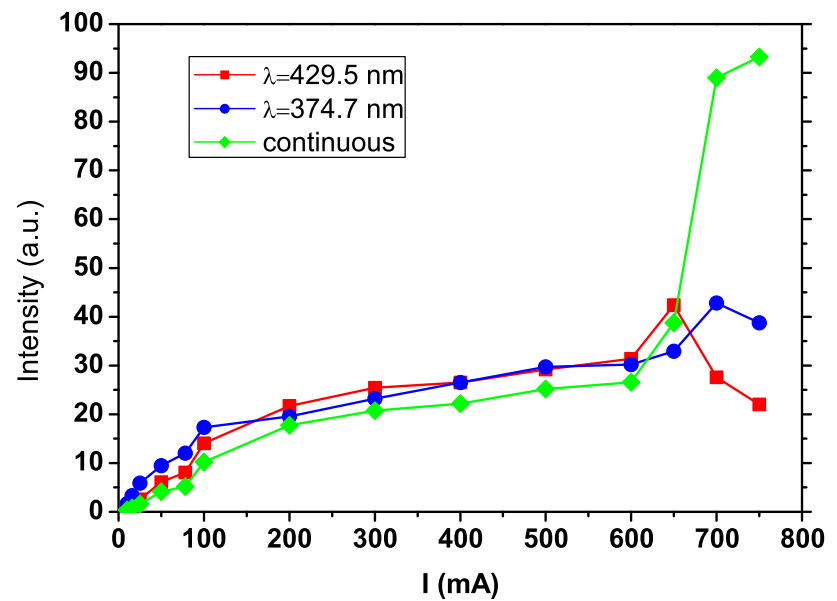

Fig. 8 Emission intensities as a function of the mean current setting

lines, until transition to arcing is achieved and the spectra approach a structureless shape above approximately $750 \mathrm{~mA}$. The reported trend is displayed in Fig. 8, where the transition could be clearly appreciated. Indeed, the continuous contribution increases quickly after the arching onset. The behavior of emission lines is more mixed. Some decays definitely, whereas others keep increasing even as at slower pace. We also observed an extended plateau region, when the increase in the mean current produces only a slight growth in the total emission, both continuous and most of the single emission lines. An interesting feature, which could barely be perceived in the spectrum (see Fig. 7), concerns the atomic oxygen line at $777 \mathrm{~nm}$. Although its intensity is almost negligible, its presence provides a hint about the potential for plasma dissociation of the airflow and for oxidation of the particles advected in it. Indeed, OES of this radical provides a foothold to assess the formation of reactive oxygen species (ROS), which are the main agents in many air plasma applications [18-20]. The trend shown reveals that it increases up to a saturation at low current settings, but it keeps increasing also after the arching transition, although its growth is somewhat linear, rather than exponential like the continuous contribution. Its intensity is reported to increase also as the pressure driving the reverse vortex flow is raised, in this case with a pace even stronger than that of the continuous. We then turn our attention to the characteristics of emission spectra when we use helium as a gas carrier in the tornado discharge. As it was stated in Introduction section, particles are easily transported and mixed in the vortex flow that sustains the discharge. They are also extracted along the central axis and could be recovered at the exit of the device. Their interaction with the discharge for only a few milliseconds avoids excessive damage by heating or erosion, which is common in arcs. Since interactions with an electrical discharge happen mainly through surface processes, the treatment of particles instead of the bulk material should show a better reactivity, due to the favorable area-to-volume ratio of the former [21]. We then studied the capability of functionalization of lignin particles by our GAT device. To this purpose, we substituted air with a non-reactive gas carrier like helium and added a bubbler in the gas line, to mix the flow with some water or HMDSO vapors. Both vapors could be dissociated in an electrical discharge and could provide radicals that could be grafted on the surface of polymers, like lignin. Again OES allows to study whether decomposition of vapor molecules could be achieved by the plasma and which radicals, among the optical active ones, are formed. Two pictures comparing the emission spectra of helium discharges with those observed after the addition of water and HMDSO vapors are shown in Figs. 9 and 10. In the case of pure helium, the first striking observation is the low level of the continuous spectrum, which remains weak even in the arching regime, which by the way is reached already at quite low mean current settings. The predominance of helium emission lines is worthy since their relative intensities could be used to gain information on the excitation temperature [22], and in cold plasma discharges, this was directly related to electron mean energy [23]. It could also be observed the presence of radicals produced in the gas phase which are possibly coming from the water vapor, probably outgassing from the pipe and device surfaces into the high purity, completely dry helium flow. This could be proved, by adding water in the bubbler of the device, in order to add a known fraction of water vapor to the flow. The OES spectra indeed look similar to previous ones with a rather strong reduction of the helium signal. Such decrease goes in favor of, in particular, the atomic hydrogen and oxygen emission lines and the large structure corresponding to the $\mathrm{OH}$ radical. So both dry and humid helium GAT could be used to provide an oxidizing set. When lignin particles were added to the flow, we observe only minor modifications in the OES spectra, together with similar electrical characteristics. Although a complete characterization of the plasma processing of lignin goes beyond the scope of this paper, we could demonstrate that grafting of functional $-\mathrm{OH}$ groups was achieved through the analysis of the Fourier-transform infrared (FTIR) 


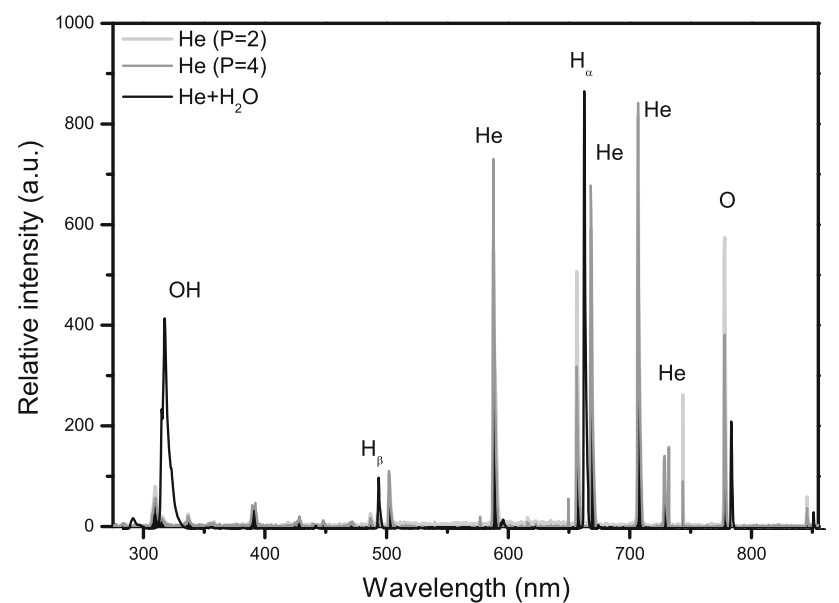

Fig. 9 Spectra of spark discharges in helium and with the addition of water vapor

spectra of the treated lignin and the modification of its water contact angle [24]. Finally, we have analyzed the capability to provide a reducing set for particle surface modification. To this purpose, we added an organic silicon compound to the flow, by charging the bubbler with HMDSO liquid. This was chosen for its high surface tension as well its reactivity in plasma gas phases $[25,26]$. A nice dissociation of the HMDSO was also observed in our GAT device. Now, the discharge spectra are completely dominated by the optical active radicals produced by the fragmentation of the HMDSO molecules, as it appears from OES spectra in Fig. 10. OES reveals the structures due to the $\mathrm{C}_{2}, \mathrm{CH}$ and atomic hydrogen emission lines, with a strong reduction of helium lines and the complete disappearance of water vapor species. The spectra resemble those emitted by spark plasmas containing methane [12]. The ratio between a few emission lines respect to a reference one of helium is shown as a function of the mean current setting in Fig. 11. It could be grasped that there is a mild increase when comparing two different excited levels of helium atoms, whereas there is a strong increase in $\mathrm{CH}$ emission line when arcing condition is reached, however, not displayed by the $\mathrm{C}_{2}$ radical. Again the addition of lignin particles to the flow produces only a minor modification of the OES spectra and discharge electrical properties. We observe also the functionalization of the polymeric particles transported in the flow from a rather significant modification of their FTIR spectra. This could be traced to atomic hydrogen surface reaction and/or deposition of $\mathrm{CH}_{x}$ or organic silicon oxide radicals on the particle surface $[27,28]$.

\section{Conclusions}

We have presented an analysis of the emission spectra of a gliding arc tornado device. Optical emission spectroscopy was used as a diagnostics tool to investigate the plasma gas phase. We show that it could

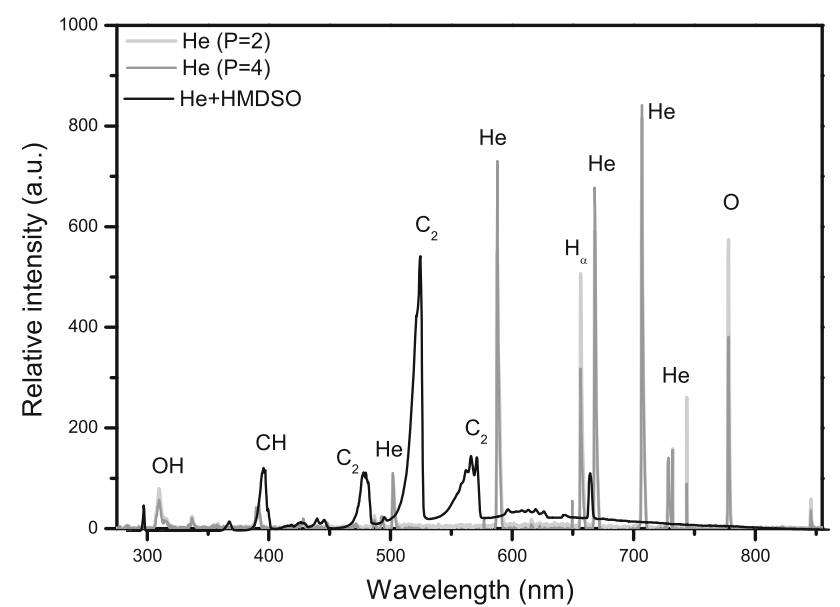

Fig. 10 Spectra of spark discharges in helium and with the addition of HMDSO vapor

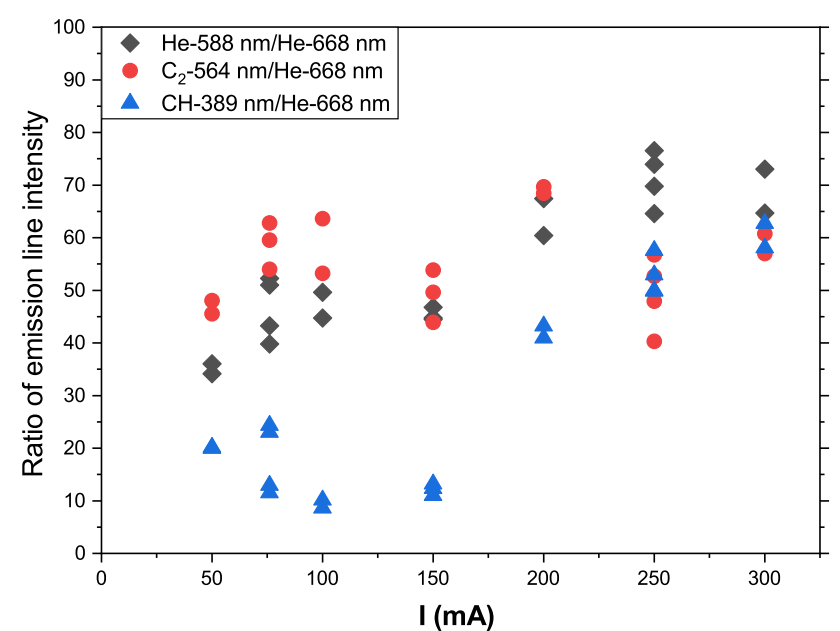

Fig. 11 Ratios of emission line intensities as a function of the mean current setting

be employed to gather information about the discharge, in particular to track the transition to arcing regime. We also studied discharge spectra in mixtures of helium with water vapor and HMDSO, which could be employed for plasma treatment of lignin particles in our device. We demonstrate that helium discharges can provide a reactive environment apt to deliver the requested surface functionalization of lignin particles because it could achieve an effective level of dissociation of suitable vapor precursors like water or HMDSO vapors. We can also conclude that optical emission spectroscopy could be used as a passive diagnostics able to monitor the conditions during plasma processing and to gather quantitative information about the production of some of the relevant reactive species.

Acknowledgements We are pleased to acknowledge the support of our technical staff at the PlasmaPrometeo Center and the funding through the POLISTE project by Cariplo Foundation. 


\section{Author contributions}

All the authors were involved in the preparation of the manuscript. All the authors have read and approved the final manuscript.

Funding Open access funding provided by Università degli Studi di Milano - Bicocca within the CRUI-CARE Agreement.

Data Availability Statement This manuscript has no associated data or the data will not be deposited. [Author's comment: The raw data will be available from the corresponding author upon reasonable request.]

Open Access This article is licensed under a Creative Commons Attribution 4.0 International License, which permits use, sharing, adaptation, distribution and reproduction in any medium or format, as long as you give appropriate credit to the original author(s) and the source, provide a link to the Creative Commons licence, and indicate if changes were made. The images or other third party material in this article are included in the article's Creative Commons licence, unless indicated otherwise in a credit line to the material. If material is not included in the article's Creative Commons licence and your intended use is not permitted by statutory regulation or exceeds the permitted use, you will need to obtain permission directly from the copyright holder. To view a copy of this licence, visit http://creativecomm ons.org/licenses/by/4.0/.

\section{References}

1. C.S. Kalra, Y.I. Choa, A. Gutsol, A. Fridman, Rev. Sci. Instr. 76, 025110 (2005)

2. J.L. Liu, H.W. Park, W.J. Chung, D.W. Park, Plasma Chem. Plasma Proc. 36, 437 (2016)

3. A.F. Bublievsky, J.C. Sagas, A.V. Gorbunov, H.S. Maciel, D.A. Bublievsky, G.B. Filho, P.T. Lacava, A.A. Halinouski, G.E. Testoni, IEEE Trans. Plasma Sci. 43. $1742(2015)$

4. S. Zanini, C. Riccardi, C. Canevali, M. Orlandi, L. Zoia, E.L. Tolppa, Bioresources 3, 995 (2008)

5. V. Passoni, C. Scarica, M. Levi, S. Turri, G. Griffini, ACS Sustain. Chem. Eng. 4, 2232 (2016)

6. S. Zanini, C. Riccardi, M. Orlandi, V. Fornara, M.P. Colombini, D.I. Donato, S. Legnaioli, V. Palleschi, Wood Sci. Technol. 42, 149 (2008)

7. A. Salanti, L. Zoia, S. Zanini, M. Orlandi, Wood Sci. Technol. 50, 117 (2016)
8. C.O. Laux, T.G. Spence, C.H. Kruger, R.N. Zare, Plasma Sources Sci. Technol. 12, 125 (2003)

9. R. Barni, S. Zanini, C. Riccardi, Vacuum 82, 217 (2008)

10. Z. Machala, M. Janda, K. Hensel, I. Jedlovsky, L. Lestinska, V. Foltin, V. Martisovits, M. Morvova, J. Mol. Spectrosc. 243, 194 (2007)

11. S. Zanini, C. Riccardi, M. Orlandi, E. Grimoldi, Vacuum 82, 290 (2008)

12. R. Barni, A. Quintini, M. Piselli, C. Riccardi, J. Appl. Phys. 103, 063302 (2008)

13. R.A. Siliprandi, H.E. Roman, R. Barni, C. Riccardi, J. Appl. Phys. 104, 063309 (2008)

14. R. Barni, I. Biganzoli, D. Tassetti, C. Riccardi, Plasma Chem. Plasma Proc. 34, 1415 (2014)

15. I. Biganzoli, R. Barni, C. Riccardi, A. Gurioli, R. Pertile, Plasma Sources Sci. Technol. 22, 025009.1-9 (2013). 22, 025009 (2013)

16. R.W.B. Pearse, A.G. Gaydon, The Identification of Molecular Spectra (Wiley, New York, 1976)

17. W.C. Martin, J.R. Fuhr, D.E. Kelleher, A. Musgrove, L. Podobedova, J. Reader, E.B. Saloman, C.J. Sansonetti, W.L. Wiese, P.J. Mohr, K. Olsen, NIST Atomic Spectra Database (NIST, Gaithersburg, 2002)

18. M.G. Kong, G. Kroesen, G. Morfill, T. Nosenko, T. Shimizu, J. van Dijk, J.L. Zimmermann, New J. Phys. 11, $115012(2009)$

19. C.A. Vasko, D.X. Liu, E.M. van Veldhuizen, F. Iza, P.J. Bruggeman, Plasma Chem. Plasma Process. 34, 1081 (2014)

20. R. Barni, P. Esena, C. Riccardi, J. Appl. Phys. 97, $073301(2005)$

21. A. Raffaele-Addamo, C. Riccardi, E. Selli, R. Barni, M. Piselli, G. Poletti, F. Orsini, B. Marcandalli, M.R. Massafra, L. Meda, Surf. Coat. Technol. 174-5, 886 (2003)

22. N.K. Podder, J.A. Johnson III., C.T. Raynor, S.D. Loch, C.P. Ballance, M.S. Pindzola, Phys. Plasmas 11, 5436 (2004)

23. M. Goto, J. Quant. Spectr. Radiat. Transf. 76, 331 (2003)

24. R. Siliprandi, S. Zanini, E. Grimoldi, F. Fumagalli, R. Barni, C. Riccardi, Plasma Chem. Plasma Process. 31, $353(2011)$

25. M. Creatore, Y. Barrell, J. Benedikt, M.C.M. Van De Sanden, Plasma Sources Sci. Technol. 15, 421 (2006)

26. P. Esena, S. Zanini, C. Riccardi, Vacuum 82, 232 (2008)

27. S. Peter, K. Graupner, D. Grambole, F. Richter, J. Appl. Phys. 102, 953 (2007)

28. S. Zanini, C. Riccardi, M. Orlandi, P. Esena, M. Tontini, M. Milani, V. Cassio, Surf. Coat. Technol. 200, 953 (2005) 